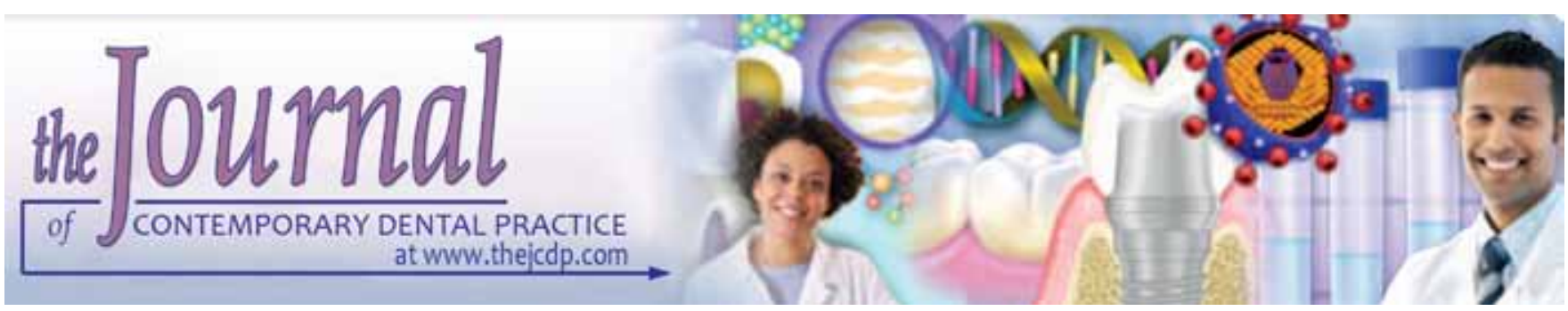

\title{
Determination of Root Canal Cleanliness by Different Irrigation Methods and Morphometric Analysis of Apical Third
}

${ }^{1}$ Fábio Heredia Seixas, ${ }^{2}$ Carlos Estrela, ${ }^{3}$ Mike Reis Bueno, ${ }^{4}$ Manoel Damião Sousa-Neto, ${ }^{5}$ Jesus Djalma Pécora

\begin{abstract}
Aim: The aim of this study was to determine the root canal area before and after the instrumentation $1 \mathrm{~mm}$ short of the apical foramen by clinical and cone beam computed tomography (CBCT) methods, and to evaluate the cleanliness of the apical region in mesiodistal flattened teeth by using optical microscopy.
\end{abstract}

Materials and methods: Forty-two human single-canal mandibular incisors were instrumented using the Free Tip Preparation technique up to three, four or five instruments from the initial. Cone beam computed tomography scans were acquired of the samples before and after root canal preparation (RCP). Irrigation was performed by conventional or hydrodynamic means, using $2.5 \%$ sodium hypochlorite. The samples were prepared for observation under an optical microscope. Images were digitally obtained, analyzed and the results were submitted to statistical analysis (two-way ANOVA complemented by Bonferroni's post-test).

Results: There was no significant difference between the studied anatomical areas with both CBCT and clinical methods. There were no differences between irrigation methods. It was verified differences between instrumentation techniques. Instrumentation with four instruments from the initial instrument determined a significant increase in the contact area when compared to preparation with three instruments, but RCP with 5 instruments did not result in a better cleanliness.

\footnotetext{
1,4,5 Department of Restorative Dentistry, Ribeirão Preto Dental School, University of São Paulo, Ribeirão Preto, SP, Brazil

${ }^{2}$ Department of Stomatologic Sciences, School of Dentistry Federal University of Goiás, Goiânia, GO, Brazil

${ }^{3}$ Department of Oral Diagnosis, School of Dentistry, University of Cuiabá, Cuiabá, MT, Brazil

Corresponding Author: Jesus Djalma Pécora, Department of Restorative Dentistry, Ribeirão Preto Dental School, University of São Paulo, Ribeirão Preto, SP, Brazil, Phone: +55-16-360024792, e-mail: jdpecora@gmail.com
}

Conclusion: The analysis with CBCT was not capable to determine the precise shape of surgical apical area comparing to the clinical method.

Clinical significance: Both the conventional and hydrodynamic irrigation techniques were not able to promote root canals debris-free. The instruments action in root canal walls was proportional to the number of instruments used from the initial apical instrument.

Keywords: Anatomic diameter, Cone beam computed tomography, Endodontic, Root canal cleaning, Rotary instrumentation.

How to cite this article: Seixas FH, Estrela C, Bueno MR, Sousa-Neto MD, Pécora JD. Determination of Root Canal Cleanliness by Different Irrigation Methods and Morphometric Analysis of Apical Third. J Contemp Dent Pract 2015;16(6):442-450.

\section{Source of support: Nil}

Conflict of interest: None

\section{INTRODUCTION}

The success of endodontic therapy is associated with the quality of root canal sanitization (RCS), which favors complete root canal filling and restoration of the lost crown structure. ${ }^{1,2}$ However, these procedures are influenced by the presence of anatomical variations (lateral, accessory and secondary canals, isthmuses, oval canals, multiple foramens and apical deltas). ${ }^{1}$ The endodontic instruments alone do not possess the capacity for adequate cleaning and require the addition of irrigating solutions. ${ }^{2-5}$

Mechanical, chemical and physical factors must be considered in root canal cleaning and shaping. Mechanical factors have great importance in root canal shaping and infection control. ${ }^{1-5}$ However, RCS does not depend only on the mechanical action of instruments but also depends on the chemical action of irrigating solutions, including dissolution of organic materials and antibacterial activity and the physical action of irrigation 
and suction. Thus, the sanitization process allows irrigating solutions to be effective in the entire root canal extension. ${ }^{1,4-8}$

Knowledge of root canal internal anatomy is directly associated with the success of endodontic therapy., ${ }^{1,6,-11}$ Apical enlargement size may be important for efficient removal of microorganisms and has been shown to influenced in the outcomes of the root canal therapy. ${ }^{2-8,10,11}$ However, studies regarding the determination of the appropriate anatomical diameter of the apical region emphasize the importance of cervical preparation. This procedure tends to assist with the correct selection of the initial instrument and leads to adequate apical third enlargement. ${ }^{2-4,6-11}$

Irrigating solutions have assumed an important role in root canal preparation (RCP). Among the available irrigating solutions, sodium hypochlorite has been widely recommended and is accepted worldwide due to its antimicrobial, deodorant, clarifying and organic tissue dissolution properties. ${ }^{1,4,5,12}$ However, it is essential to consider the penetration depth of the irrigating cannula, the frequency of irrigation, the contact area, the abundance of irrigation and the concentration of irrigant solutions are all of equal importance as the choice of irrigating solution to obtain effective sanitization. 4,5,12

Technological advances during the last decade have provided new devices for agitation and mechanisms for irrigating solutions. These technologies can promote removal of the smear layer and debris in root canal walls during root canal preparation. ${ }^{4,5,8,12}$ Recently, a new irrigation device developed from automatic hydrodynamic irrigation technology utilizing pressure and suction was introduced to the market (RinsEndo ${ }^{\circledR}$ ). Several studies have evaluated this new mechanism. ${ }^{13-19}$

Ideally, root canal instrumentation techniques should result in contact with every wall, and thus promote cleanliness and removal of microorganisms. Therefore, it is necessary to determine which instrument will be the first to touch the walls. This promotes contact between the instruments and root canal walls and can help establish how many instruments should be used during surgery. Furthermore, it is necessary to determine the appropriate irrigation method to achieve root canal cleanliness, particularly for teeth with mesiodistal root flattening.

The aim of this study was to determine the area of the root canal before and after instrumentation was performed $1 \mathrm{~mm}$ short of the apical foramen (working length, WL) by clinical and tomographic analysis. We also aimed to evaluate the cleanliness of the apical region of root canals with mesiodistal flattening by optical microscopy.

\section{MATERIALS AND METHODS}

\section{Teeth Selection}

Forty-two human mandible incisors ex vivo with completely formed apices obtained from the dental stock of the Dentistry School of Ribeirão Preto of São Paulo University were used in this study. The exclusion criteria included teeth with two canals in the root, filled root canals, incomplete rhizogenesis, calcified root canals, root canals with severe curvature, and a tooth height lower than 19 and higher than $21 \mathrm{~mm}$.

The selected teeth were stored in a thymol $0.1 \%$ solution at $9^{\circ} \mathrm{C}$ until the time of use. The specimens were washed in running water for 2 hours to remove any thymol residue. Next, chemically activated acrylic resin that was $11 \times 20 \mathrm{~cm}$ in dimension was used to pin the teeth and to obtain a standard to perform the initial and final imaging exams. Next, crowns were added to the teeth inside silicone, distributing the specimens in three rows with seven teeth each, with their axes (buccallingual and mesiodistal) parallel.

Perforations using a spherical carbide burr \#6 (FKG, Brazil) driven by a micromotor (Dabi Atlante, Brazil) were performed in an acrylic plate $3 \mathrm{~mm}$ in depth and diameter. Three perforations were performed in one side of the plate, 2 on one side and one on the other side. Two more perforations were performed in a diagonal pattern on the other side of the plate to guide the tomographic exams and the identification of the teeth. These perforations were filled with gutta-percha to provide radiopaque images.

\section{Cone Beam Computed Tomography Analysis}

Our method utilized cone beam computed tomography imaging (CBCT, I-CAT's 3D ${ }^{\circledR}$, Dental Imaging System, Salt Lake City, USA). The initial CBCT exam was performed using root transversal slices (axial scans) to measure the apical anatomical region of the samples $1 \mathrm{~mm}$ short of the apical foramen (in the WL). The measurements were in millimeters by the reading sequence. The measurements were properly recorded and the identified teeth were then used for later comparison with the clinical method of initial apical instrument selection. At the same time the measurement was taken, a line of $5 \mathrm{~mm}$ for later calibration of the measurement tool was created. An acrylic plate was fixed in two bases of red wax measuring $2 \times 5 \mathrm{~cm}$, so that the teeth were immersed but suspended in the colorless plastic $12 \times 20 \mathrm{~cm}$ container filled with water. The container with the samples was placed on the tomograph table which was suitable for research, where the X-rays beams of the tomograph could reach the entire 
sample. After performing the initial tomograph exam, access surgery was performed and a k-type instrument \#10 was inserted until it was visualized in the apical foramen. The working length was achieved by removing $1 \mathrm{~mm}$ from this measurement.

\section{Root Canal Preparation}

Once the working length (WL) was obtained, LA Axxess burrs (SybronEndo, Glendora, California, USA) (20/0.06 and 35/0.06) driven by a no-reduction hand piece (Dabi Atlante, Ribeirão Preto, SP, Brazil), at a speed of 10,000 rpm were used to eliminate cervical and medial interference. After the root canals were irrigated with a $2.5 \%$ sodium hypochlorite solution, the initial apical instrument (IAI) was selected. This procedure was performed with the aid of K-flex type files $25 \mathrm{~mm}$ in length (Densell ${ }^{\circledR}$, Sweden) that were a better fit for the apical anatomical diameter of each tooth. This instrument and the measurement of the diameter of the instrument were registered for later comparison with the area obtained by the tomographic method.

After this stage, the teeth were divided in two groups of 21 teeth each and distributed by the irrigation type: group 1 received conventional irrigation (luer lock syringe and needle), while group 2 received hydrodynamic irrigation (RinsEndo ${ }^{\circledR}$, Durr Dental, Germany).

Each group was divided in three subgroups according to the number of instruments used after the IAI. For subgroup 1 three instruments were used after IAI, for subgroup 2 four instruments were used and, for subgroup 3, five instruments were used.

The root canal instrumentation was performed using the Free Tip Preparation technique (20) utilizing RT-Files (Densell ${ }^{\circledR}$, Sweden) and Quantec series ${ }^{\circledR}$ (SybronEndo, USA) driven by an electrical handpiece EndoEst ${ }^{\circledR}$ (Geosoft ${ }^{\circledR}$, Russia) at a constant speed of $250 \mathrm{rpm}$ in the WL. Root canal preparation (RCP) was performed by the file corresponding to the anatomical diameter found clinically by the algorithm 20/0.02; 20/0.04; 25/0.02; 25/0.04; 30/0.02; 30/0.04; $30 / 0.06$. The procedure was finished after use of three instruments after the IAI was used on the first row of teeth in the acrylic plate, after use of four instruments after the IAI in the second row and after use of five instruments after the IAI in the third row with $(35,40,45$, 50, 55/0.02) RT-Files ${ }^{\circledR}$ (Densell ${ }^{\circledR}$, Sweden) and \#60/0.02 Quantec series ${ }^{\circledR}$ (SybronEndo, USA).

\section{Irrigation Technique}

After each change in instrument, the root canal was irrigated with $2 \mathrm{ml}$ of a $2.5 \%$ sodium hypochlorite solution, dispensed and properly titrated in the Endodontics
Research Laboratory, with a final irrigation of $2 \mathrm{ml}$ of the same solution and $2 \mathrm{ml}$ of $17 \%$ EDTA. Each group of teeth received the same irrigating solution for root canal preparation. The difference was in the method used to perform the irrigation-suction process.

In group 1, conventional irrigation with a Luer lock $5 \mathrm{ml}$ syringe (Ultradent ${ }^{\circledR}$ Products, USA), irrigation needles Endo-Eze Irrigator Tips ${ }^{\circledR}$ (27 gauges diameter) (Ultradent ${ }^{\circledR}$ Products Inc, South Jordan, EUA) with lateral openings, and a conventional suction with a vacuum pump (Ciclone ${ }^{\circledR}$, Dabi Atlant, Ribeirão Preto, SP, Brazil) was used. In group 2, hydrodynamic automatic irrigation was used, utilizing pressure with the RinsEndo ${ }^{\circledR}$ device (Dürr Dental, Germany) with a 30 gauge diameter needle designed for irrigation with a lateral opening of $7 \mathrm{~mm}$ (RinsEndo ${ }^{\circledR}$, Dürr Dental, Germany), which is a superior device for using a syringe with irrigating solution and suction similar to that used for group I. The system was plugged into the handpiece (Dabi Atlante, Brazil) and driven pneumatically. The device contained a plastic piece over the needle to avoid reflux of the irrigating solution towards the patient and/or the dental professional, as well as a lateral opening to introduce the suction cannula. The RCPs were performed by a single professional who is a specialist in endodontics with clinical experience in the endodontic system tested and the different irrigation methods utilized.

\section{Analysis of Surgical Diameter by CBCT}

After RCP was performed, new CBCT imaging was performed using the same protocol described for the initial tomographic exam. The second imaging aimed to compare the measurements of the surgical apical areas by clinical and CBCT methods in the WL.

\section{Determination of Surgical Area Diameter}

Analysis of topograms were performed using the software I-CAT Vision ${ }^{\circledR}$ (Dental Imaging System, Salt Lake City, USA) to measure the apical area in the WL, which was obtained by performing an apical-coronary scan of each tooth until the apical foramen was visible. From this point, $1 \mathrm{~mm}$ towards the coronary region was measured to establish the exact analysis point. Then, the image was selected and identified with the respective number of the tooth, and the 'distance tool' created a $5 \mathrm{~mm}$ line directly below the sample as a calibration standard for the measurement tool.

The 'area tool' of the software Imagetool ${ }^{\circledR} 3.0$ for Windows ${ }^{\circledR}$ (University of Texas Health Science Center, San Antonio, TX, USA) was used to measure the sample area. This measurement was expressed in square 
millimeters and was obtained for the specimens before and after the biomechanical root canal preparations and subsequently registered for comparison and statistical analysis. The images of the specimens were assessed, and the anatomical and surgical apical areas in the WL were measured by three specialists in endodontics. After the RCP and final CBCT imaging was complete, the specimens were removed from the acrylic plate and prepared for histological analysis. The samples were immersed and properly identified in individual containers containing a $10 \%$ formalin solution, were stored for 12 hours, and then, were washed and placed in individual containers containing an aqueous solution of $10 \%$ trichloroacetic acid that was replaced every 24 hours over 5 days for decalcification.

After decalcification, the specimens were washed and neutralized with a $4 \%$ sodium sulfate solution for 15 minutes. The specimens were then washed in running water for 15 minutes. The crowns, cervical and medium thirds were dismissed. The apical thirds were submitted to decalcification, cleared and embedded in paraffin blocks.

Subsequently, serial sections were performed with $5 \mu \mathrm{m}$ of thickness, dismissing the apical millimeter. The plates were mounted and staining with hematoxylin and eosin was performed.

Ten slices of each specimen were selected for morphometric study. The first 50 slices were dismissed to evaluate the region closer to the working length. The next slice (slice 51) was taken for morphometry. From the 51 slices, 10 slices were successively selected from each root. An optical microscope Eclipse E 600 (Nikon, Shinagawa-ku, Tokyo, Japan) with a $4 \times / 0.13$ ocular and a $10 \times / 25$ magnification lens was used, for a final $40 \times$ magnification. The images were captured using the software Movie Maker ${ }^{\circledR}$ (Microsoft, USA).

\section{Determination of Areas with Debris}

The image was analyzed using the software Imagetool ${ }^{\circledR}$ 3.0 for Windows (University of Texas Health Science Center, San Antonio, TX, USA). The 'area tool' measured the total area of the root canal and the area that contained debris. It was then possible to calculate the debris area percentage of the root canal by the rule of thirds.

\section{Instrument Action Perimeter Determination}

The determination of instrument perimeter action in transversal slices of the root canal was performed using the same process and software described above. The area of the root canal walls not contacted by the instrument was measured. This measurement was subtracted from the total working length to determine the area in which the root canal was effective. The instrument action in the root canal wall was determined based on the following observations: surface regularity, abrupt change in the wall continuity and partial or total removal of the predentin layer.

\section{Histological Analysis Criteria}

Histological analysis was performed by determining the presence or absence of debris and pulp remnants in the root canal walls, the visualization of untouched areas, evaluation of the pre-dentin layer, and the regularity of root canal walls. The results were registered in a file that was properly completed with the observed data. The resulting anatomical and surgical areas exposed by the clinical method and by CBCT imaging were compared, as were the levels of cleanliness achieved by the different irrigation methods and instrumentation diameters. Statistical analysis was performed using the software GrafPad ${ }^{\circledR}$ Prism, version 5.0 (GraphPad Software Inc, USA).

\section{RESULTS}

The values obtained by measurement of the area of the WL (1 $\mathrm{mm}$ smaller than the apical foramen) by CBCT before (anatomical) and after (surgical) RCP are displayed in Table 1. The measurements of the WL obtained by the clinical method were determined, theoretically, in the circular area occupied by the file, and the results are described in Table 1. The data in Table 1 were grouped to perform descriptive statistics and to determine the nature of the sampling distribution. The D'Agostino and Pearson normality test indicated a Gaussian distribution of the sample, which allowed for performance of a parametric test to statistically analyze the results. These results were submitted to two-way variance analysis (ANOVA) complemented by Bonferroni's Test to perform multiple comparisons between the groups. Variance analysis revealed a significant difference between the two measurements of the surgical apical area $(p<0.001)$, and higher measurements were observed with the clinical method. However, the analysis of the anatomical apical area did not differ significantly between the different measurement methods $(p>0.05)$. Table 2 displays the results obtained by optical microscopy analysis of the clean areas of the apical third for the specimens irrigated by conventional and hydrodynamic techniques.

The optical microscopy results regarding the cleanliness of the root canals were submitted to normality tests (D'Agostino and Pearson) to determine the nature of sample distribution. The square transformation of 
Table 1: Apical área corresponding to working length by using CBCT and clinical method $(\mathrm{mm})$

\begin{tabular}{|c|c|c|c|c|c|c|c|c|c|c|c|}
\hline \multicolumn{12}{|c|}{ Apical area determined by $C B C T$} \\
\hline \multicolumn{6}{|c|}{ Anatomical } & \multicolumn{6}{|c|}{ Surgical } \\
\hline 0.041 & 0.028 & 0.067 & 0.057 & 0.019 & 0.079 & 0.128 & 0.075 & 0.166 & 0.164 & 0.091 & 0.220 \\
\hline 0.24 & 0.035 & 0.020 & 0.027 & 0.044 & 0.037 & 0.098 & 0.156 & 0.123 & 0.076 & 0.112 & 0.105 \\
\hline 0.020 & 0.016 & 0.060 & 0.044 & 0.025 & 0.102 & 0.148 & 0.175 & 0.161 & 0.114 & 0.151 & 0.141 \\
\hline 0.067 & 0.082 & 0.086 & 0.035 & 0.030 & 0.024 & 0.096 & 0.073 & 0.167 & 0.110 & 0.104 & 0.176 \\
\hline 0.021 & 0.047 & 0.011 & 0.024 & 0.045 & 0.039 & 0.099 & 0.101 & 0.080 & 0.124 & 0.113 & 0.107 \\
\hline 0.015 & 0.029 & 0.092 & 0.026 & 0.142 & 0.104 & 0.071 & 0.166 & 0.141 & 0.090 & 0.123 & 0.095 \\
\hline 0.043 & 0.082 & 0.046 & 0.028 & 0.040 & 0.075 & 0.088 & 0.113 & 0.128 & 0.062 & 0.115 & 0.197 \\
\hline \multicolumn{6}{|c|}{ Mean and standard deviation $0.047 \pm 0.029$} & \multicolumn{6}{|c|}{ Mean and standard deviation $0.122 \pm 0.037$} \\
\hline \multicolumn{12}{|c|}{ Apical area determined by clinical method } \\
\hline \multicolumn{6}{|c|}{ Anatomical } & \multicolumn{6}{|c|}{ Surgical } \\
\hline 0.071 & 0.126 & 0.049 & 0.096 & 0.049 & 0.049 & 0.126 & 0.159 & 0.238 & 0.159 & 0.126 & 0.238 \\
\hline 0.071 & 0.049 & 0.071 & 0.071 & 0.049 & 0.049 & 0.159 & 0.196 & 0.196 & 0.126 & 0.126 & 0.159 \\
\hline 0.049 & 0.071 & 0.071 & 0.071 & 0.031 & 0.071 & 0.126 & 0.159 & 0.238 & 0.159 & 0.159 & 0.283 \\
\hline 0.071 & 0.049 & 0.049 & 0.096 & 0.071 & 0.031 & 0.126 & 0.196 & 0.238 & 0.126 & 0.159 & 0.159 \\
\hline 0.031 & 0.031 & 0.031 & 0.071 & 0.031 & 0.071 & 0.096 & 0.159 & 0.159 & 0.096 & 0.159 & 0.196 \\
\hline 0.049 & 0.031 & 0.031 & 0.049 & 0.049 & 0.031 & 0.096 & 0.126 & 0.196 & 0.159 & 0.126 & 0.238 \\
\hline 0.031 & 0.049 & 0.031 & 0.031 & 0.049 & 0.049 & 0.096 & 0.126 & 0.159 & 0.096 & 0.196 & 0.283 \\
\hline
\end{tabular}

Table 2: Values of the debris-free areas after RCP (\%)

\begin{tabular}{|c|c|c|c|c|c|}
\hline \multicolumn{3}{|c|}{ Conventional irrigation } & \multicolumn{3}{|c|}{ Hydrodynamic irrigation } \\
\hline$|A|+3$ & $|A|+4$ & $|A|+5$ & $|A|+3$ & $|A|+4$ & $|A|+5$ \\
\hline 100.00 & 77.06 & 89.22 & 84.23 & 84.92 & - \\
\hline- & 98.70 & 99.20 & - & 97.52 & 98.56 \\
\hline 96.04 & 97.37 & 85.32 & 64.33 & 97.63 & 100.00 \\
\hline 69.95 & 87.19 & - & 100.00 & 94.55 & 100.00 \\
\hline 77.64 & 92.81 & 97.63 & 100.00 & - & 76.66 \\
\hline 93.65 & 84.60 & 63.21 & 78.36 & 65.82 & 98.40 \\
\hline 53.56 & 95.98 & 100.00 & 53.25 & 100.00 & 97.99 \\
\hline $81.81 \pm 18.05$ & $90.53 \pm 7.92$ & $89.10 \pm 13.99$ & $80.03 \pm 18.88$ & $90.07 \pm 13.01$ & $95.27 \pm 9.16$ \\
\hline
\end{tabular}

The results with trace refer to specimens that were lost in the histotechnical preparation; IAI: Initial apical instrument

Table 3: Percentage of dentin in the apical area by sectional cross considering the performance of the instrument (original values)

\begin{tabular}{|c|c|c|c|c|c|}
\hline \multicolumn{3}{|c|}{ Conventional irrigation } & \multicolumn{3}{|c|}{ Hydrodynamic irrigation } \\
\hline$|A|+3$ & $|A|+4$ & $\mid A I+5$ & $|A|+3$ & $|A|+4$ & $|A|+5$ \\
\hline 4.7 & 75.32 & 49.47 & 59.39 & 58.82 & - \\
\hline- & 18.84 & 91.18 & - & 73.29 & 90 \\
\hline 20.89 & 58.33 & 67.86 & 2.50 & 88.98 & 100 \\
\hline 47.66 & 63.50 & - & 26.35 & 95.27 & 72.85 \\
\hline 40.26 & 77.39 & 93.71 & 43.11 & - & 82.01 \\
\hline 19.39 & 65.25 & 96.73 & 40 & 64.44 & 93.09 \\
\hline 27.48 & 78.94 & 93.01 & 16.36 & 97.50 & 93.60 \\
\hline $26.73 \pm 15.45$ & $62.51 \pm 20.77$ & $81.99 \pm 19.06$ & $31.28 \pm 20.40$ & $79.71 \pm 16.46$ & $88.59 \pm 9.88$ \\
\hline
\end{tabular}

The results with trace refer to specimens that were lost in the histotechnical preparation; IAI: Initial apical instrument

the original data was used to approximate the values to a Gaussian curve. The adjusted results were submitted to a two-way ANOVA analysis. The statistical analysis revealed no significant differences between the types of irrigation tested ( $p=0.73$ ) or between the instruments $(p=0.14)$, which was justification for not performing Bonferroni's test. Thus, the percentage of cleanliness obtained in the apical region did not differ significantly between teeth instrumented and irrigated with the conventional technique $v s$ the hydrodynamic technique.
The results corresponding to the percentage of instrument action extension in the root canal walls are listed in Table 3. The transformed results were submitted to a two-way ANOVA analysis. While the irrigation method did not have a significant impact on the instrument action extension in root canal walls ( $p=0.11$, statistical analysis revealed a difference between the tested groups with regards to the instrumentation technique ( $\mathrm{p}<0.001)$. The biomechanical preparation using the four instruments in addition to the IAI resulted in an 
Table 4: Analysis of apical geometry of root canals by CBCT images (\%)

\begin{tabular}{lll}
\hline & Anatomical (initial) & Surgical (final) \\
\hline Flattened & 52.3 & 66.6 \\
Circular & 42 & 28.7 \\
Irregular & 4.7 & 4.7 \\
\hline
\end{tabular}

action area that was significantly greater than the same preparation with only three instruments in addition to the IAI. However, preparation with five instruments in addition to the IAI did not significantly enhance the root canal preparation in the apical third of the tooth when compared to preparation with 4 instruments in addition to the IAI ( $p>0.05)$.

Tables 4 and 5 display the apical geometry analysis of the root canals in the working length of the mandibular incisors with the percentages of each shape. These results were consistent regardless of the method utilized. There was a higher prevalence of elliptical shapes in the apical region of the root canals. Figures $1 \mathrm{~A}$ to $\mathrm{H}$ display photomicrographs of specimens from the apical region that are representative of the tested groups.

\section{DISCUSSION}

Various strategies of cleaning, sanitization, shaping and sealing of the root canals have been suggested to obtain better outcomes after endodontic therapy. ${ }^{1,3-9,13-20}$ The complexity of the internal anatomy of the root canal system and its variations with regards to the number and shape deserve special attention, particularly in cases of root flattening. In such cases, some areas are difficult to access, which prevents efficient cleaning and shaping. Mandibular incisors were selected for this experiment because of the requirement for special attention during RCP of these teeth.

Knowledge of the anatomical root canal apical diameter in the WL is indispensable for efficient cleaning of root canal walls, as the appropriate IAI is determined from this dimension. The most accepted method to clinically determine the anatomical diameter involved tactile insertion of the instrument that best fits in the root canal walls. In vitro determination of the anatomical apical diameter has been previously discussed. 2,3,6,7,10,11 Better outcomes in IAI selection are more reliable when cervical preparation has been previously performed.

The precise determination of the anatomic diameter in vivo still has limitations. Radiographic exam presents limited details for measuring the diameter of root canals because it is a two-dimensional image of a threedimensional structure. With regards to the search for a superior method, our study utilized CBCT, which has the advantage of three-dimensional visualization
Table 5: Analysis of apical geometry of root canals by histological and CBCT method (\%)

\begin{tabular}{lll}
\hline & Surgical (histological) & Surgical (CBCT) \\
\hline Flattened & 56.6 & 66.6 \\
Circular & 37 & 28.7 \\
Irregular & 5.4 & 4.7 \\
\hline
\end{tabular}

of the apical area and the internal morphology of root canals. ${ }^{21,22}$ Our study did not find a significant difference between tomographic and clinical methods with regards to determination of the anatomical apical area in the working length. However, when comparing the measurements of instrumented root canal areas (surgical diameter) using CBCT scans of the apical areas of the final instruments using the clinical method, a significant difference was found, with higher values resulting from use of the clinical method. These findings are likely due to the limitations that are inherent to obtaining adequate visualization of small areas, such as the space of the root canal in the apical third as well as the effects of the secondary radiation from radiographic process, which generated a penumbra area around the structures. These aspects made precise visualization of the root canal wall limits difficult, even with the use of the filters present in the software (I'Cat vision). Analysis was, thus, subjective and imprecise. In contrast, analysis of the diameter and configuration of the internal anatomy of human teeth using computerized microtomograph technology allows for the production of an image with superior definition. ${ }^{23,24}$

Other important factor for successful outcomes of endodontic therapy is root canal cleanliness and sanitization. Our study used optical microscopy to assess the quantity of debris in the apical third of root canals prepared by NiTi rotatory instruments, with three different apical shapings, and irrigated by $2.5 \%$ sodium hypochlorite, by hydrodynamic (RinsEndo ${ }^{\circledR}$ ) or using conventional methods. The results showed that there is no significant difference between the two irrigation techniques. Both hydrodynamic and conventional methods yielded similar results in removing debris from root canals. Vivan et $\mathrm{al}^{19}$ found similar results. However, Caron et $\mathrm{al}^{15}$ and Rödig et $\mathrm{al}^{18}$ reported that hydrodynamic automated irrigation with RinsEndo ${ }^{\circledR}$ yielded inferior results when compared to other irrigating methods, which contradicts the studies by McGill et al ${ }^{17}$ and Hauser et al. ${ }^{16}$

The hypothesis that the larger the apical preparation, and the better the cleanliness was not confirmed in our study. The level of cleanliness obtained by different enlargement levels did not result in debris-free canals, without significant differences between the methods. 

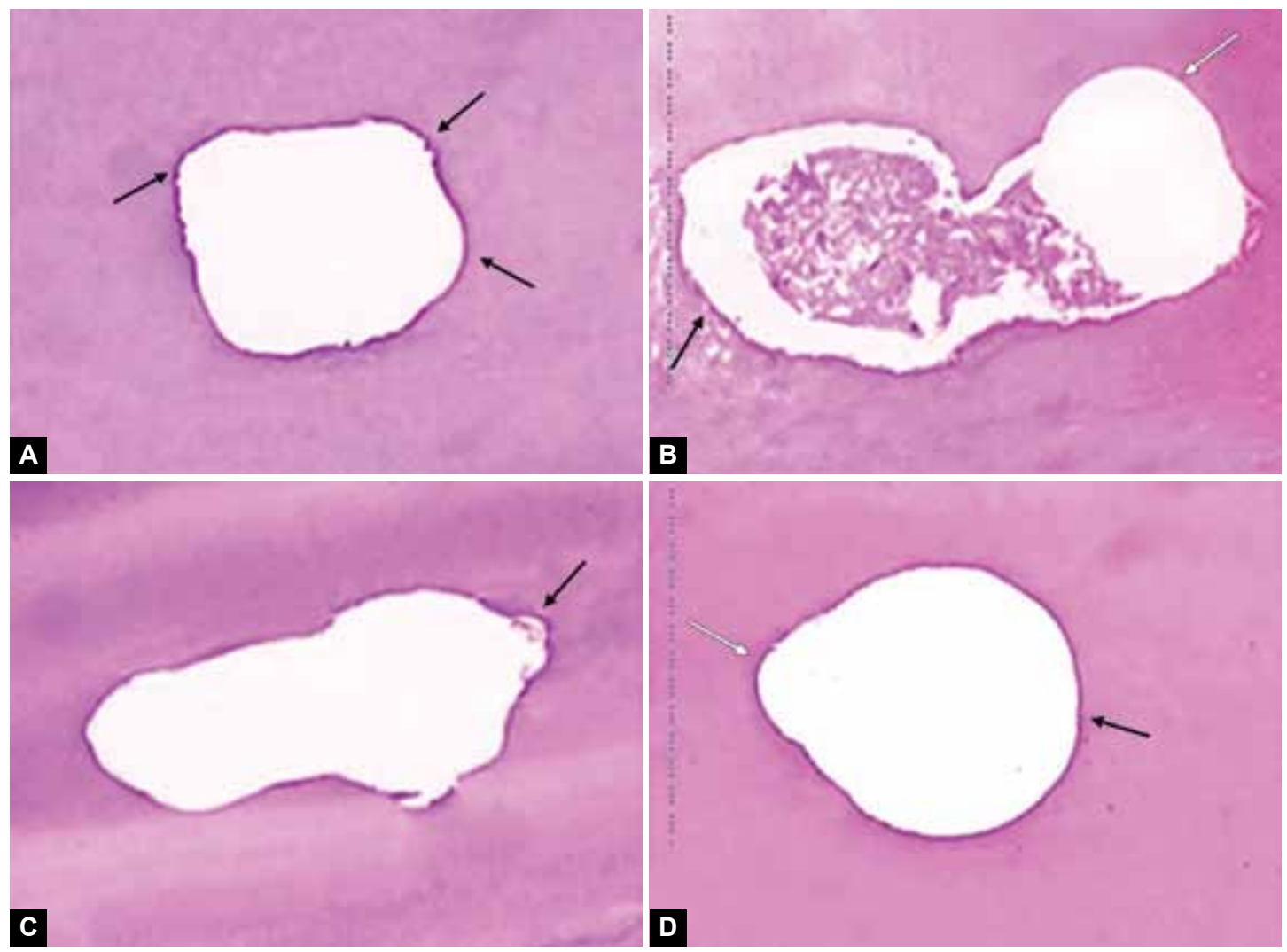

C
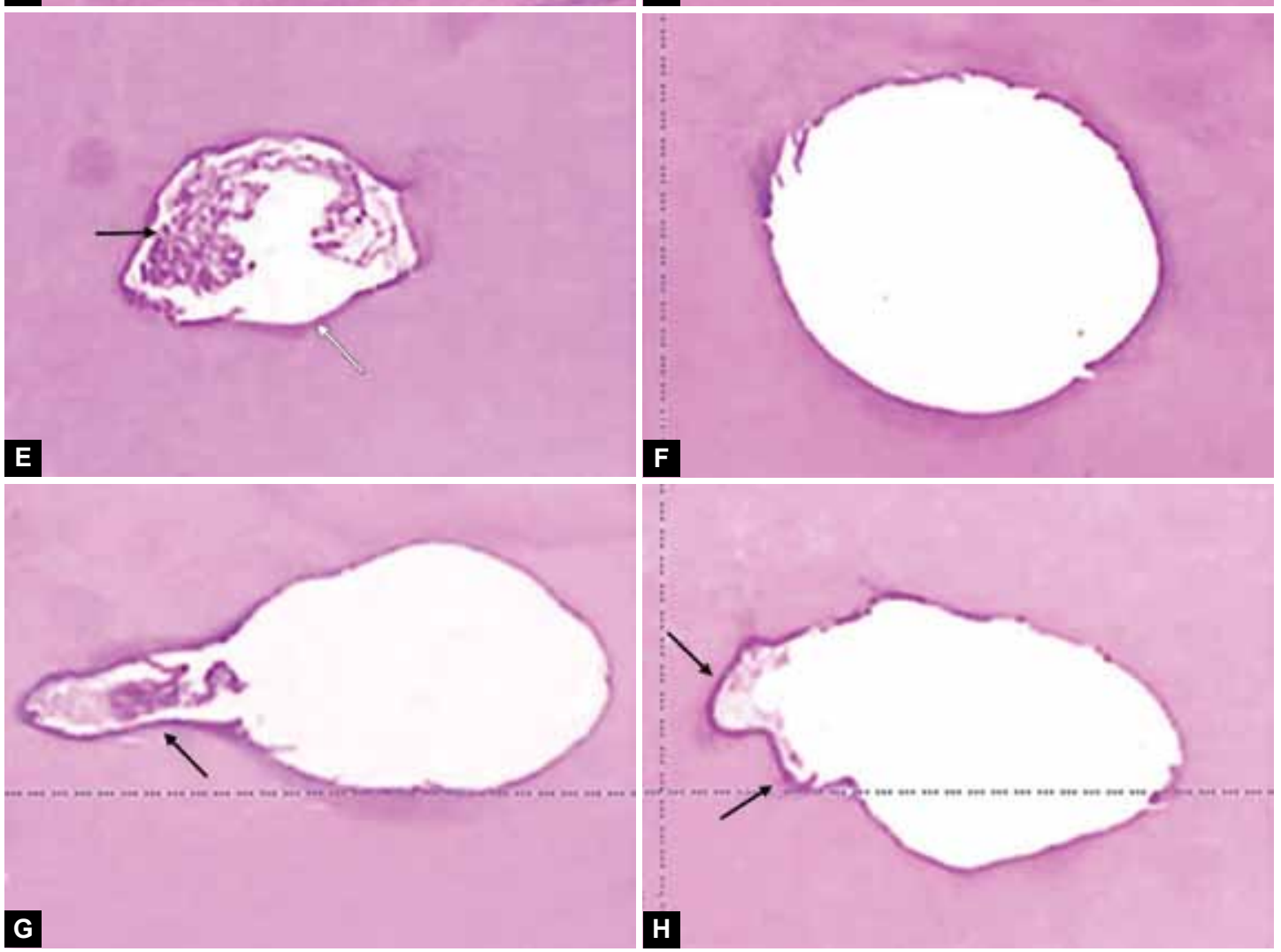

G

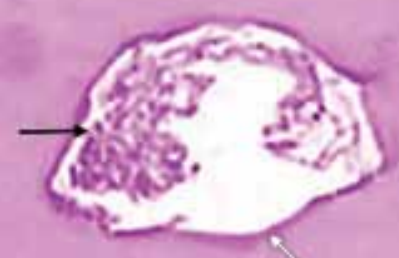

E

Figs 1A to H: Group 1: Apical region (A) Root canal unprepared (arrows), (3 instruments above IAl, absence of debris, (B) flat root canal prepared with three instruments above IAI showing debris; area without action of instrument (black arrow), and area that the instrument prepared the root canal (white arrow), (C) area with debris, (black arrow) prepared with four instruments above IAI, without action of instrument in the walls, (D) walls prepared (black arrow) and unprepared (white arrow); RCP with five instruments above IAI, and absence of debris (hematoxylin and eosin; original magnification, 40x). Group 2: Apical region (E) root canal with pronounced flattening, unprepared (arrow), (3 instruments above IAI, presence of debris) (black arrow); (F) root canal prepared with three instruments above IAI showing debris; area with action of instrument, $(G)$ area with much debris (arrows) prepared with three instruments above IAI, without action of instrument in the walls, $(\mathrm{H}) \mathrm{RCP}$ with five instruments above IAI, and absence of debris (hematoxylin and eosin: original magnification $40 x$ ). 
Histological analysis showed that instruments did not promote an effective preparation for every root canal wall, leaving untouched areas and failing to obtain effective RCS. This fact was emphasized by Kereks and Tronstand. ${ }^{25,26}$ In our study, several root canal walls remained untouched, showing that complete cleanliness was not achieved, even with use of five instruments in addition to the IAI.

However, histological analysis of the root canal walls that remained untouched by instruments revealed a significant difference with different levels of apical enlargement. Root canal preparation with four instruments in addition to the IAI resulted in a significantly superior area of action in comparison with the same method with only three instruments in addition to the IAI. The use of four instruments in addition to the IAI was superior with regards to instrumentation of root canal walls and removal of the predentin layer. On the other hand, there was not a significant difference when comparing the results of using either four or five instruments in addition to the IAI. This indicates the need to use a larger apical preparation to achieve better results in instrumentation of all root canal walls in the WL. Previous studies showed that certain root morphology characteristics (such as root flatness) may contribute to post-procedure infection, as the instruments may not touch all of the root canal walls, and thus do not produce the expected root canal cleaning results.

\section{CONCLUSION}

The analysis of apical areas with CBCT was unable to determine the precise shape of the surgical apical area when compared with the clinical method. There was no significant difference between the studied anatomical areas using either the tomographic or the clinical method. The irrigation techniques did not result in debris-free root canals. The instruments action in root canal walls was directly proportional to the number of instruments used above IAI.

\section{ACKNOWLEDGMENT}

The authors thank Ulisses Campregher (Dürr Dental of Brazil) and Eduardo Gdikian (Trading Lohcus Health) for their support with instruments and apparatus used in this study.

\section{REFERENCES}

1. Nair PNR, Henry S, Cano V, Vera J. Microbial status of apical root canal system of human mandibular first molars with primary apical periodontitis after one-visit-endodontic treatment. Oral Surg Oral Med Oral Pathol Oral Radiol Endod 2005;99:231-252.
2. Peters OA. Current challenges and concepts in the preparation of root canal systems: a review. J Endod 2004;30:559-567.

3. Paqué $F$, Zehnder M, Marending M. Apical fit of initial $\mathrm{K}$-files in maxillary molars assessed by micro-computed tomography. Int Endod J 2012;43:328-335.

4. Estrela CRA, Estrela C, Reis C, Bammann LL, Pécora JD. Control of microorganisms in vitro by endodontic irrigants. Braz Dent J 2003;14:187-192.

5. Siqueira JJR, Alves FRF, Almeida BM, Oliveira JCM, Rôças IN. Ability of chemomechanical preparation with either rotary instruments or self-adjusting file to disinfect oval-shaped root canals. J Endod 2010;36:1860-1865.

6. Pécora JD, Capelli A, Guerisoli DMZ, Spano JCE, Estrela C. Influence of cervical preparation on apical file size determination. Int Endod J 2005;38:430-435.

7. Marchesan MA, Arruda MP, Silva-Sousa YTC, Saquy PC, Pecora JD, Sousa-Neto MD. Morphometrical analysis of cleaning capacity using nickel-titanium rotary instrumentation associated with irrigating solutions in mesio-distal flattened root canals. J Appl Oral Sci 2003;11:55-59.

8. Yared GM, Bou Dagher FE. Influence of apical enlargement on bacterial infection during treatment of apical periodontitis. J Endod 1994;20:535-537.

9. Steven JC, Sigurdsson A, Ørstavik D, Trope M. the effectiveness of increased apical enlargement in reducing intracanal bacteria. J Endod 2002;28:779-783.

10. Wu MK, Roris A, Barkis D, Wesselink PR. Prevalence and extent of long oval shape of canals in the apical third. Oral Surg, Oral Med, Oral Pathol, Oral Radiol Endod 2000;89: 739-743.

11. Wu MK, Barkis D, Roris A, Wesselink PR. Does the first file to bind correspond to the diameter of the canal in the apical region? Int Endod J 2002;35:264-267.

12. Estrela C, Estrela CRA, Barbin EL, Spanó JC, Marchesan MA, Pécora JD. Mechanism of action of sodium hypochlorite. Braz Dent J 2002;13:113-117.

13. Gu L, Kim JR, Ling J, Choi KK, Pasheley DH, Tay FR. Review of contemporary irrigant agitation techniques and device. J Endod 2009;35:791-804.

14. Metzger Z, Teperovich E, Zary R, Cohen R, Hof R. The Selfadjusting file (SAF). Part 1: Respecting the root canal anatomy: a new concept of endodontic files and its implementation. J Endod 2010;36:679-990.

15. Caron G, Nham K, Bronnec F, Machtou P. Effectiveness of different final irrigant activation protocols on smear layer removal in curved canals. J Endod 2010;36:1361-1366.

16. Hauser V, Braun A, Frentzen M. Penetration depth of a dye marker into dentine using a novel hydrodynamic system (RinsEndo). Int Endod J 2007;40:644-652.

17. Mcgill S, Gulabivala K, Mordam N, NG YL. The efficacy of dynamic irrigation using a commercially available system (RinsEndo) determined by removal of a collagen bio-molecular film from an ex vivo model. Int Endod J 2008;41:602-608.

18. Rödig T, Sedghi M, Konietschke F, Lange K, Ziebolz D, Hülsmann M. Efficacy of syringe irrigation, RinsEndo and passive ultrasonic irrigation in removing debris from irregularities in root canals with different apical sizes. Int Endod J 2010;43:581-589. 
19. Vivan RR, Bortolo MV, Duarte MAH, Moraes IG, TanomaruFilho M, Bramante CM. Scanning electron microscopy analysis of RinsEndo system and conventional irrigation for débris removal. Braz Dent J 2010;21:305-309.

20. Pécora JD, Capelli A, Seixas FH, Maechesan MA, Guerisoli DMZ. Rotary Biomechanics: reality or future? Rev Ass Paul Cir Dent 2002;56:4-6.

21. Durack C, Patel S. Cone beam computed tomography in endodontics. Braz Dent J 2012;23:179-191.

22. Estrela C, Bueno MR, Leles CR, Azevedo B, Azevedo JR. Accuracy of cone beam computed tomography and panoramic radiography for the detection of apical periodontitis. J Endod 2008;34:273-279.
23. Peters OA,LaibA,RüegssegerP,BarbakowF.Three-dimensional analysis of root canal geometry by high-resolution computed tomography. J Dent Res 2000;79:1405-1409.

24. Somma F, Leoni D, Plotino G, Grande NM, Plasschaert A. Root canal morphology of the mesiobuccal root of maxilary first molars: a micro-computed tomographic analysis. Int Endod J 2009;42:165-174.

25. Kerekes K, Tronstand L. Morphometric observations on the root canals of human anterior teeth. J Endod 1977;3:24-29.

26. Ghorbanzadeh S, Loodaricheh SA, Samizade S, Zadsirjan S. Irrigants in endodontic treatment. Int J Contemp Dent Med Rev 2015; 2015: Article ID: 030515. DOI: 10.15713/ins. ijcdmr.78 